\title{
Hepatocystin Is Not Secreted in Cyst Fluid of Hepatocystin Mutant Polycystic Liver Patients
}

\author{
Esmé Waanders, ${ }^{*}{ }^{\dagger}$ Anke L. L. Lameris, ${ }^{\dagger}$ Huub J. M. Op den Camp, ${ }^{\ddagger}$ Wendy Pluk, ${ }^{\$}$ \\ Jolein Gloerich, ${ }^{\S}$ Simon P. Strijk," and Joost P. H. Drenth ${ }^{\dagger}$
}

\begin{abstract}
Department of Gastroenterology and Hepatology, Radboud University Nijmegen Medical Center, P.O. Box 9101, 6500 HB Nijmegen, The Netherlands, Department of Microbiology, Institute for Water and Wetland Research, Radboud University Nijmegen, Toernooiveld 1, 6525 ED Nijmegen, The Netherlands, Nijmegen Proteomics Facility, Radboud University Nijmegen Medical Center, P.O. Box 9101, 6500 HB Nijmegen, The Netherlands, and Department of Radiology, Radboud University Nijmegen Medical Center, P.O. Box 9101,
\end{abstract} 6500 HB Nijmegen, The Netherlands

Received January 14, 2008

\begin{abstract}
Autosomal dominant polycystic liver disease (PCLD) is characterized by multiple liver cysts and is caused by mutations in PRKCSH (hepatocystin). Mechanisms of cystogenesis are unknown, but previous studies have shown that hepatocystin is secreted in vitro. The goal of this study was to determine the fate of hepatocystin in vivo. Using immunoprecipitation, we determined that mutant hepatocystin is secreted from both apical and basolateral cell surface of MDCK cells stably transfected with mutant hepatocystin. Analysis of 60 cyst fluid samples from polycystic livers using Western blot, MALDI-TOF MS or nLC-MS/ MS did not detect hepatocystin in liver cyst fluid. We did identify 163 ubiquitous serum proteins. No paracrine or autocrine factors were recognized. Although cyst fluids vary greatly in protein concentration, a PCLD specific protein pattern was not established. In conclusion, hepatocystin is not secreted in PCLD liver cyst fluid, suggesting that mutant hepatocystin is either not produced or degraded intracellularly. PCLD cysts develop from intralobular bile ductules and cyst fluid mainly contains common serum proteins comparable to that of other polycystic diseases.
\end{abstract}

Keywords: PCLD • liver cyst fluid • PRKCSH • hepatocystin • protein profile • MALDI-TOF MS • $\mathrm{nLC}-\mathrm{MS} / \mathrm{MS}$

\section{Introduction}

Polycystic liver disease (PCLD) is an autosomal dominant inherited condition characterized by numerous fluid filled cysts in the liver, without renal involvement. ${ }^{1}$ The main differential diagnosis is autosomal dominant polycystic kidney disease (ADPKD). Here, the predominant feature is the presence of polycystic kidneys, though up to $80 \%$ of the patients develop hepatic cysts as the primary extrarenal manifestation. ${ }^{2}$

PCLD is associated with mutations in PRKCSH ${ }^{3,4}$ This gene encodes hepatocystin, a protein that is involved in the folding of glycoproteins in the endoplasmic reticulum. It serves as the noncatalytic $\beta$-subunit of $\alpha$-glucosidase, an enzyme that cleaves the two innermost glucoses from the mannose tree of glycoproteins. ${ }^{5}$ This releases the glycoprotein from calreticulin/ calnexin and makes it available for translocation to the Golgi.

\footnotetext{
* To whom correspondence should be addressed. Department of Gastroenterology and Hepatology 455, Radboud University Nijmegen Medical Center, P.O. Box 9101, 6500 HB Nijmegen, The Netherlands. Phone, +31 24 3616520; fax, +31 24 3540103; e-mail, e.waanders@mdl.umcn.nl.

${ }^{\dagger}$ Department of Gastroenterology and Hepatology, Radboud University Nijmegen Medical Center.

${ }^{\ddagger}$ Department of Microbiology, Institute for Water and Wetland Research, Radboud University Nijmegen.

${ }^{\S}$ Nijmegen Proteomics Facility, Radboud University Nijmegen Medical Center.

"Department of Radiology, Radboud University Nijmegen Medical Center.
}

This mechanism represents the quality control for correct folding of glycoproteins in the ER (reviewed in ref 6).

Most hepatocystin mutations associated with PCLD lead to premature termination of translation producing a truncated protein. Transfection studies in HeLa cells have shown that mutant, but not wild-type PRKCSH, results in a protein that does not assemble with the $\alpha$-subunit, loses retainment in the ER and is secreted into the medium. ${ }^{7}$ It is unclear whether these in vitro findings reflect the situation in PCLD, and whether hepatocystin is secreted in vivo.

Recent developments in mass spectrometry technologies have enabled analysis of differential proteomics, such as the comparison between normal and disease status. This allows identification of protein markers that may serve to identify a specific physiological or pathological state. We used singledimension SDS-PAGE for protein separation in combination with high-throughput techniques for protein identification in liver cyst fluids. We performed proteomic analysis on a platform of matrix-assisted laser desorption/ionization-time-of-flight mass spectrometry (MALDI-TOF MS) and nanoscale liquid chromatography tandem mass spectrometry (nLC-MS/MS).

This study was designed with the main aim to determine whether hepatocystin is secreted in cyst fluid in vivo. We hypothesized that similar to in vitro experiments, (mutant) hepatocystin is secreted into hepatic cysts of patients carrying 
a mutant PRKCSH allele. Second, we set out to identify substantially abundant proteins in cyst fluid from PCLD patients in order to obtain a unique protein signature. In addition, we hoped to identify autocrine and paracrine factors that stimulate cyst formation and growth. The results may help to clarify the mechanisms that drive the hepatic cystogenesis and may aid in the identification of biomarkers of the disease.

\section{Experimental Procedures}

Development of Cell Lines. Madin-Darby canine kidney (MDCK) cells were cultured in Dulbecco's Modified Eagle Medium (DMEM) supplemented with $10 \%$ fetal bovine serum, penicillin $100 \mathrm{U} / \mathrm{mL}$ and streptomycin $100 \mathrm{U} / \mathrm{mL}$. We transfected the cells with eukaryote expression vectors containing wild-type and mutated $P R K C S H^{7}$ using Fugene reagent (Roche, Indianapolis, IN). Transfected clones expressing the introduced genes were selected using G418 resistance. Stably transfected MDCK cells were grown on an Anopore filter with a pore size of $0.02 \mu \mathrm{m}$ (Nalge Nunc International, Rochester, NY). Medium was collected on the apical and basolateral side of the membrane after 3, 6, 9, and $12 \mathrm{~h}$ and subsequently used for immunoprecipitation experiments.

Immuoprecipitation. Aliquots with equal protein concentration were precipitated with $30 \mu \mathrm{L}$ of anti-HA monoclonal antibody (clone HA.11, Covance, Richmond, CA) bound to protein G/agarose beads (Santa Cruz Biotechnology, Santa Cruz, CA) for $16 \mathrm{~h}$ at $4{ }^{\circ} \mathrm{C}$. Next, samples were centrifuged (3 min, 2000g, $4{ }^{\circ} \mathrm{C}$ ), washed twice with $1 \mathrm{~mL}$ of lysis buffer containing $0.1 \%$ Triton X-100, and subsequently washed once with $0.5 \mathrm{~mL}$ of PBS. Samples were then analyzed on Western blot using anti-HA antibody.

Western Blot. Proteins resolved by SDS-PAGE were transferred onto a Protran nitrocellulose membrane (Whatman Inc., Florham Park, NJ). Membranes were blocked with 5\% low-fat milk and incubated overnight with primary antibodies, directed against Hemagglutinin (HA)-tag (clone HA.11, Covance, Richmond, CA) or hepatocystin (BD Transduction Laboratories, San Jose, CA), followed by incubation with appropriate secondary antibodies labeled with horseradish peroxidase (HRP). Secondary antibodies were visualized using ECL Western blotting detection reagents (GE Healthcare Limited, Buckinghamshire, U.K.) and Hyperfilm MP (Amersham Biosciences UK Ltd., Buckinghamshire, U.K.).

Patients and Samples. PCLD was diagnosed when (1) more than 20 liver cysts were seen on abdominal computer tomography and the formal ultrasonographic criteria for ADPKD were not applicable ${ }^{8}$ or (2) mutations were found in PRKCSH. We collected 60 cyst fluid samples from 25 patients who underwent percutaneous cyst aspiration or laparoscopic cyst fenestration. The patient group consisted of 16 PCLD patients (50 samples, all female), 3 ADPKD patients (3 samples, 2 female/ 1 male) and 6 patients with isolated liver cysts who did not meet the PCLD or ADPKD criteria (7 samples, 4 female/2 male). Samples were frozen in aliquots to prevent recurrent thawing and stored at $-20{ }^{\circ} \mathrm{C}$.

Genotyping. Patients included in this study were screened for PCLD mutations by extracting genomic DNA from peripheral blood leukocytes (Gentra Systems, Minneapolis, MN) and genotyping for $\mathrm{PRKCSH}$ as described earlier. ${ }^{9}$ From the 16 PCLD patients, 7 patients showed a mutation in $P R K C S H$. The patients with isolated liver cysts were all wild-type for $P R K C S H$.
Routine Chemistry. Total protein concentration and albumin concentration was determined in all fluids using an Aeroset 2.0 automated analyzer (Abbott Laboratories, Abbott Park, IL).

Protein Profiling. All cyst fluid samples were subjected to SDS-PAGE analysis. The samples were albumin-depleted and subsequently concentrated to prevent disruption of experiments by high albumin concentrations. Albumin was removed from cyst fluids using ProteoExtract Albumin removal kit (Calbiochem, Darmstadt, Germany) and the samples were concentrated using YM3 Microcon centrifugal filter devices (Millipore, Billerica, MA) according to manufacturer's instructions. After separation on a $12 \%$ polyacrylamide gel electrophoresis, samples were either processed for Western blot analysis or proteins were in-gel-digested with trypsin followed by matrix-assisted laser desorption/ionization time-of-flight mass spectrometry (MALDI-TOF MS) for identification of proteins.

In-Gel Trypsin Digestion. SDS-PAGE gels were fixed in $12 \%$ TCA, stained with Coomassie Brilliant Blue G250 and destained in $20 \%$ methanol $/ 7 \%$ acetic acid. Gels were then carefully sealed in $23 \%$ ammonium sulfate and stored at $4{ }^{\circ} \mathrm{C}$ until further use. Each of the protein bands visible on SDS-PAGE gels was analyzed in 10 different samples. Spots of interest were cut from the gels (approximately $1.5 \mathrm{~mm}^{3}$ ) using a scalpel or a pipet. Gel pieces were washed with $25 \mathrm{mM}$ ammonium bicarbonate and dehydrated with increasing concentrations of acetonitrile. Next, gel pieces were rehydrated on ice with $\sim 15 \mathrm{ng} / \mu \mathrm{L}$ Trypsin Gold (Promega, Madison, WI) in $25 \mathrm{mM}$ ammoniumbicarbonate/ $5 \mathrm{mM} n$-octyl- $\beta$-D-glucopyranoside. Excess trypsin solution was removed, and pieces were covered with $25 \mathrm{mM}$ ammoniumbicarbonate $/ 5 \mathrm{mM} n$-octyl- $\beta$-D-glucopyranoside and incubated overnight at $37^{\circ} \mathrm{C}$. Peptides were extracted using $50 \%$ acetonitrile/ $0.5 \%$ trifluoroacetic acid (TFA) $/ 5 \mathrm{mM} n$-octyl- $\beta$-Dglucopyranoside. After $1 \mathrm{~h}$ incubation and $2 \mathrm{~min}$ sonification, the liquid was transferred to a new tube, and extracts were dried using a vacuum centrifuge.

MALDI-TOF MS. Samples were dissolved in $0.1 \%$ TFA, spotted on an MTP ground steel plate, covered with a matrix solution containing $20 \mathrm{mg} / \mathrm{mL}$ 4-hydroxy- $\alpha$-cyanocinnamic acid in $50 \%$ acetonitrile $/ 0.05 \%$ TFA, and allowed to air-dry. Series of eight samples were spotted around one external calibration mixture. This calibration mix contained $1.26 \mathrm{mM}$ bradikinin, $1.26 \mathrm{mM}$ P14R, $0.19 \mathrm{mM}$ angiotensin and $0.95 \mathrm{mM}$ ACTH in matrix solution. After protein spotting, the sample plate was transferred into the mass spectrometer (Bruker Biflex III MALDI-TOF MS, Bruker Daltonics Inc., Billerica, MA) and spectra were obtained in the reflectron mode. All relevant peaks seen on the MALDI-TOF MS spectrum were manually annotated as their monoisotopic peptide peaks and the respective $\mathrm{m} / \mathrm{z}$ values were stored in a peak search list using the X-TOF program (Version 5.1.0, Bruker Daltonics, Billerica, MA). The search list representing the protein peptide mass fingerprint was compared to the Swiss-Prot database (version 20040730) using BioTools (Version 2.01, Bruker Daltonics, Billerica, MA) and the MASCOT search algorithm (Version 1.6, Matrix Science, Inc., Boston, MA). Search parameters were set to look for proteins from Homo sapiens only, with methionine oxidation as a possible modification and allowing 1 trypsin miscleavage. Protein identifications were assigned a Mowse score calculated by $-10 \log (P)$, were $P$ is the probability that the observed match is a random event. ${ }^{10} \mathrm{~A}$ Mowse score greater than 53 with a protein mass tolerance $\leq 0.2$ Da was considered significant $(p<0.05)$. Resulting hits were scrutinized based on their Mowse 
score, sequence coverage, and the similarity between theoretical and experimental molecular weight obtained from gel electrophoresis. Keratin identifications were excluded since they are likely to be contaminants in MALDI-TOF MS experiments. ${ }^{11}$

nLC-MS/MS. A pool of 5 cyst fluid samples from different patients was analyzed using nanoflow liquid chromatography linear ion trap Fourier transform-ion cyclotron resonance mass spectrometry (nLC-LIT FT-ICR MS). The proteins were separated on a $12 \%$ SDS-PAGE gel. The gel was divided into 5 pieces (front-23 kDa, 23-30 kDa, 30-50 kDa, 50-70 kDa, $70 \mathrm{kDa}$-high end), which were subsequently in-gel-reduced with $10 \mathrm{mM}$ dithiotreitol, alkylated with $50 \mathrm{mM}$ iodoacetamide and digested with trypsin. Prior to $\mathrm{nLC}-\mathrm{MS} / \mathrm{MS}$ analysis, samples were purified and desalted using Stage tips. ${ }^{12}$ Peptides were analyzed using the nLC LTQ FTMS (Thermo Scientific, Waltham, MA) equipment of the Nijmegen Proteomics Facility (NPF, www. proteomicsnijmegen.nl) and RAW spectrum files were converted with the aid of DTAsupercharge (http://msquant. sourceforge.net/) into a Mascot generic peak list. Peptides and proteins were identified using the Mascot search program (Version 2.1.03, Matrix Science Inc., Boston, MA, USA) and the NCBInr database (version 20070212, www.ncbi.nlm.nih.gov) using $H$. sapiens taxonomy. The search algorithm was set to perform a tryptic search allowing 2 miscleavages and searched for carbamidomethyl modifications on cysteine residues and possible oxidation of methionine and deamidation of glutamine and asparagine, with a mass accuracy of $20 \mathrm{ppm}$ for the ICR cell and 0.8 Da for the LIT of the instrument. False-discovery rates (FDR) were estimated by Mascot searches performed against the reversed protein database using the criteria mentioned above. To validate the protein hits resulting from Mascot, the files were reanalyzed using PROVALT. ${ }^{13}$ This automated program takes large MS data sets and reorganizes them by grouping together peptides according to their predicted matching protein. FDR calculations by PROVALT provide a reasonable balance between the number of correct and incorrect protein assignments. In this study, the FDR was set at $1 \%$. PROVALT clusters proteins together in homologous groups. From each group, only the protein identified with the most peptides per protein was taken into account for further analyses.

The annotation of protein cellular localization and biological function was performed using gene ontology (GO) biological process and the GO cellular component terms. ${ }^{14} \mathrm{GO}$ analysis was performed using ProteinCenter (Proxeon Biosystems A/S, Odense Denmark, www.proxeon.com).

\section{Results}

Mutated Hepatocystin Is Secreted by MDCK Cells. To determine whether hepatocystin can be secreted in cyst fluid, we first analyzed on which side of the polarized epithelial cell hepatocystin is secreted. To this end, we stably transfected MDCK cells with epitope-tagged wild-type and mutant PRKCSH. Immunoprecipitation and Western blot analysis confirmed expression of full-length (wild-type) and truncated (mutant) hepatocystin by these cell lines (Figure 1A). Next, we cultured the cells on a membrane and analyzed medium on the apical and basolateral side of the cells. We found that truncated hepatocystin is secreted into both the apical and basolateral medium. Secretion was both substantial and rapid, detectable only $3 \mathrm{~h}$ after changing the medium. In contrast,

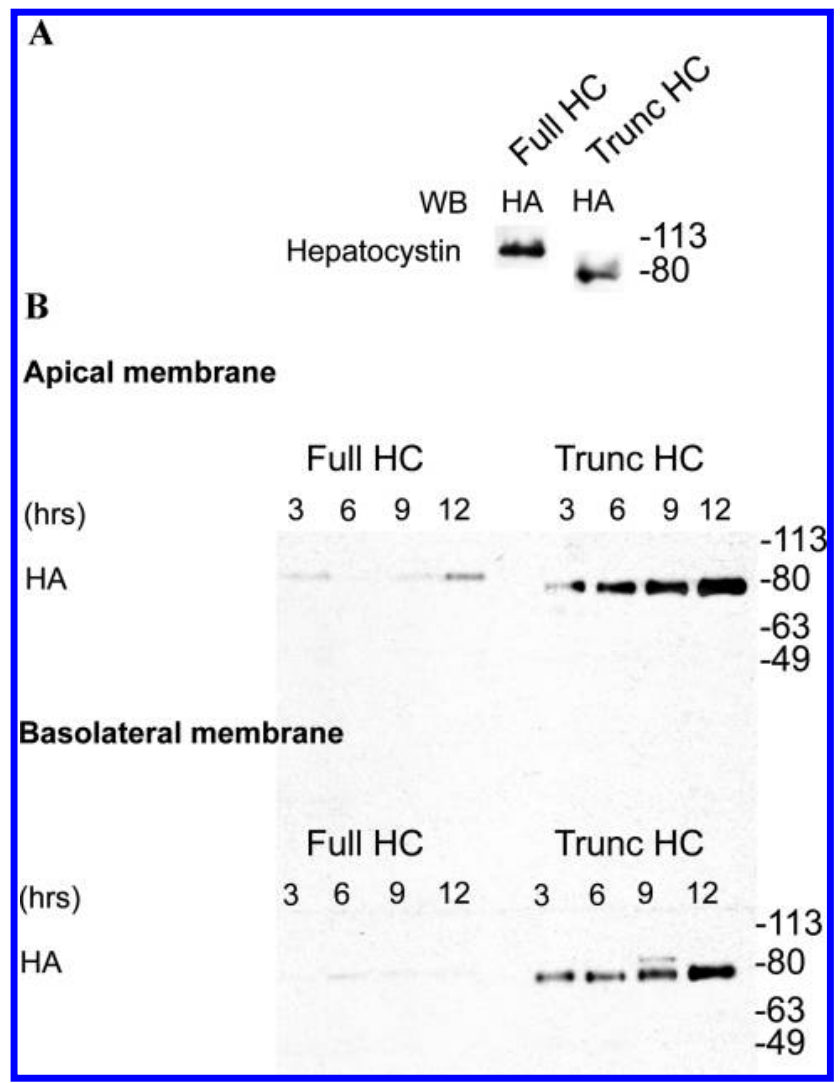

Figure 1. Immunoblot analysis of hepatocystin secretion by MDCK cells. (A) HA-tagged wild-type (full-length) and mutant (truncated) hepatocystin are expressed in stably transfected MDCK cells. Introduced hepatocystin was detected on immunoblot using anti-HA tag antibody. (B) Mutated hepatocystin is secreted form the apical and the basolateral side of MDCK cells. Stably transfected MDCK cells were grown on an Anopore membrane. After 3, 6, 9, or $12 \mathrm{~h}$, medium was collected from the apical and the basolateral side and immunoprecipitated using an antibody against HA-tag. Protein was visualized on immunoblot with an anti-HA-tag antibody. Notice that full-length (wildtype) hepatocystin is not secreted by the MDCK cells.

full-length hepatocystin was retained in the cells. The presence of the HA-tag after $12 \mathrm{~h}$ probably represents loss through cell lysis.

General Chemistry of Human Cyst Fluids. A total of 60 liver cyst fluids were included in this study (16 PCLD patients, 50 samples; 3 ADPKD patients, 3 samples; 6 patients with isolated liver cysts, 7 samples). We detected 7 heterozygous $P R K C S H$ mutation carriers among 16 tested PCLD patients, while the remaining patients were wild-type for $P R K C S H$. Total protein and albumin concentrations were determined in all fluid samples. Both parameters were within the range of normal values in serum, but showed great variability. The mean total protein content of hepatic cysts was $25.2 \mathrm{~g} / \mathrm{L}$ (range $0.2-84.4$ $\mathrm{g} / \mathrm{L}$ ), while mean albumin value was $12.9 \mathrm{~g} / \mathrm{L}$ (range $0-44 \mathrm{~g} / \mathrm{L}$ ). There were large differences between patients, but also between individual cysts obtained from the same patient. We observed no differences between PCLD, ADPKD and isolated liver cyst content.

Hepatocystin Is Absent in PCLD Liver Cyst Fluid. To study the presence of hepatocystin in liver cyst fluid, we subjected 17 PCLD cyst fluid samples (6 PRKCSH wild-type, 11 PRKCSH mutants) to Western blot analysis followed by immunostaining for hepatocystin. We failed to detect mutant hepatocystin in 


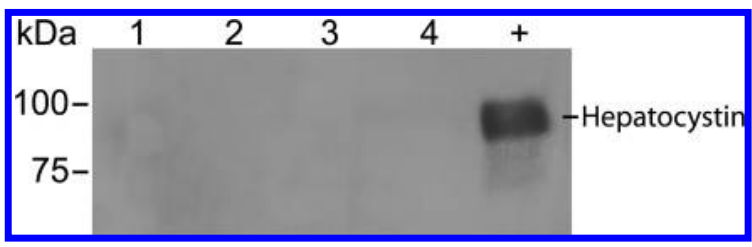

Figure 2. Western blot analysis of hepatocystin in cyst fluids. A representative image is shown of four PCLD cyst fluids (numbered lanes) and a positive control (+). Albumin-depleted and concentrated samples were subjected to Western blot analysis followed by immunostaining of hepatocystin. No hepatocystin was detected in the cyst fluids. We used cholangiocyte whole cell lysate for positive control. Molecular mass is indicated on the left $(\mathrm{kDa})$.

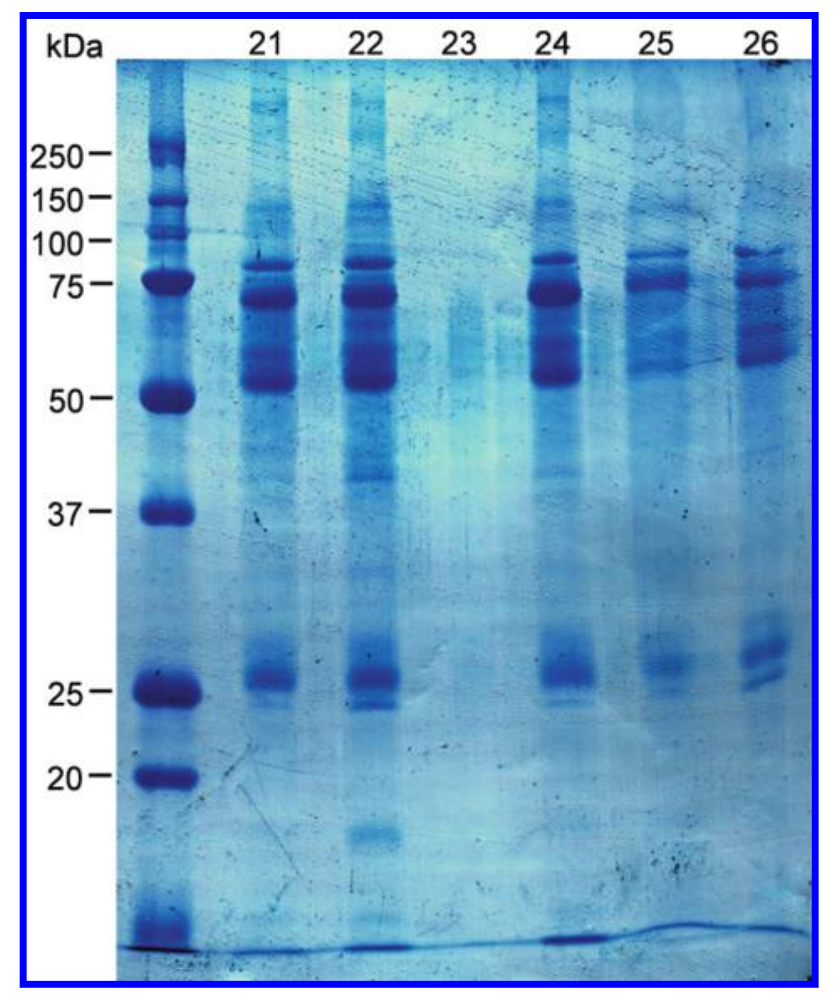

Figure 3. Protein profile of PCLD cyst fluid. A representative image is shown of six PCLD cyst fluids (numbered lanes). Cyst fluid samples were albumin-depleted and subsequently concentrated to prevent disruption by high albumin concentrations. After SDS PAGE electrophoresis, proteins were visualized using Coomassie Brilliant Blue. Notice highly similar and serum-like protein profiles for all patients. Molecular mass is indicated on the left $(\mathrm{kDa})$.

fluids from PRKCSH mutation carriers. Likewise, none of the cyst fluids demonstrated wild-type hepatocystin staining, indicating the absence of hepatocystin in these samples (Figure 2).

MALDI-TOF MS Analysis. Next, we determined the protein profile of 60 cyst fluids (50 PCLD, 3 ADPKD and 7 isolated liver cysts) by one-dimensional SDS-PAGE gel electrophoresis and protein staining with Coomassie Brilliant Blue (Figure 3). We compared the protein profiles with profiles from three healthy control sera. The cyst fluid profiles revealed highly similar images consistent with a serum-like pattern. We observed no staining pattern unique to fluids derived from either group.

Electrophoresis of cyst fluids revealed 25 unique protein bands. To limit variation, we handpicked each band 10 times from the gels. Samples were subsequently analyzed using in- gel trypsin digestion and MALDI-TOF MS. We identified a total of 10 proteins (Table 1). We detected transferrin and ferritin, both involved in iron transport, and apolipoprotein A-1 and transthyretin, which participate in cholesterol and thyroxine transport, respectively. Furthermore, we identified albumin, $\alpha$-1-antitrypsin, and complement C3 in addition to fibronectin and fibrinogen. One sample contained hemoglobin indicating that the cyst had bled. All proteins identified are commonly present in serum. All identifications were confirmed with nLC-MS/MS analysis (see below), although $\alpha$-1-antitrypsin was not a first hit in its protein group. Hepatocystin was not detected in our samples.

Nano-LC MS/MS. Finally, we analyzed a pool of 5 PCLD liver cyst fluids with one-dimensional SDS-PAGE gel electrophoresis followed by nLC-MS/MS. In addition to the 10 proteins already detected by MALDI-TOF MS, nLC-MS/MS demonstrated the presence of 153 unique proteins, when likely contaminants such as keratins were omitted (Supplementary Table 1, the first hit in each group is highlighted in red, likely contaminants are listed in gray). Again, hepatocystin was not present.

Classification of the identified proteins according to their Gene Ontology (GO) terms showed that of the 163 identified proteins 119 proteins are involved in metabolism (73\%), 67 proteins are involved in regulation of biological processes (41\%), and 63 proteins are involved in defense response (39\%) (Figure 4A). Next, we classified proteins according to their cellular component GO annotation. This revealed that most identified proteins were extracellular (127 proteins, $78 \%$ ), or associated with membranes (52 proteins, 32\%). Also, 36 proteins were associated with protein complexes (22\%) (Figure 4B).

\section{Discussion}

Here we show that (1) mutant hepatocystin is secreted from MDCK cells on the apical as well as on the basolateral side; (2) liver cyst fluids from PCLD, ADPKD and isolated liver cysts vary greatly in total protein and albumin concentration, but show similarity in protein profile when visualized on SDS-PAGE gel electrophoresis; (3) none of the fluids analyzed contain hepatocystin; and (4) the 163 proteins identified by MALDI/TOF MS and nLC-MS/MS are mainly extracellular or membrane bound and involved in metabolism and regulation of biological processes.

Previous research has shown that mutant hepatocystin can be secreted from HeLa cells. ${ }^{7}$ In this study, we confirm this finding and we show in addition that mutant hepatocystin is secreted on the apical as well as on the basolateral side of MDCK cells. This suggests that cysts from patients harboring a PRKCSH mutation theoretically might contain mutant hepatocystin.

We used 3 different techniques to analyze hepatocystin presence in human liver cyst fluid, but failed to identify hepatocystin in our samples. This discrepancy can be explained two-fold. First, the in vitro experiments comprise artificial introduction and overexpression of protein, and might not have been the correct reflection of the in vivo setting. Second, highabundance proteins such as albumin, which constitutes $50 \%$ of protein content in cyst fluids, are a disturbing factor in either of the used techniques. We depleted all samples from albumin, but samples were not completely dealbuminized as shown by both MALDI-TOF MS and nLC-MS/MS analysis. Therefore, would hepatocystin be bound to albumin, it would have been detected as well by these techniques. Finally, we performed a 
Table 1. Overview of Proteins Identified by MALDI-TOF MS Analysis ${ }^{a}$

\begin{tabular}{|c|c|c|c|c|c|c|}
\hline protein & $\begin{array}{l}\text { Swiss-Prot } \\
\text { accession no. }\end{array}$ & $\begin{array}{l}\text { average Mowse } \\
\text { score }\end{array}$ & no. hits & $\begin{array}{l}\text { no. peptides } \\
\text { matched }\end{array}$ & $\begin{array}{l}\text { sequence } \\
\text { coverage (\%) }\end{array}$ & $\begin{array}{l}\text { nLC-MS/MS } \\
\text { confirmation }\end{array}$ \\
\hline Fibronectin & P02751 & 95 & $3 / 10$ & $22 / 42$ & 19 & Yes \\
\hline Serotransferrin & P02787 & 109 & $2 / 10$ & $18 / 29$ & 28 & Yes \\
\hline Serum albumin & P02768 & 121 & $8 / 10$ & $16 / 21$ & 34 & Yes \\
\hline$\alpha$-1-antitrypsin & P01009 & 117 & $10 / 10$ & $15 / 40$ & 56 & Yes \\
\hline Fibrinogen $\beta$ chain & P02675 & 90 & $6 / 10$ & $21 / 34$ & 41 & Yes \\
\hline Transthyretin & P02766 & 65 & $2 / 10$ & $5 / 22$ & 74 & Yes \\
\hline Hemoglobin $\beta$ chain & P68871 & 74 & $1 / 10$ & $6 / 24$ & 51 & Yes \\
\hline
\end{tabular}

${ }^{a}$ No. hits represents the number of identifications of the particular protein in relation to the number of samples analyzed. No. peptides matched are based on the annotation in the hit with the highest Mowse score. Total sequence coverage is calculated from the peptides matched in all hits for a specific protein.

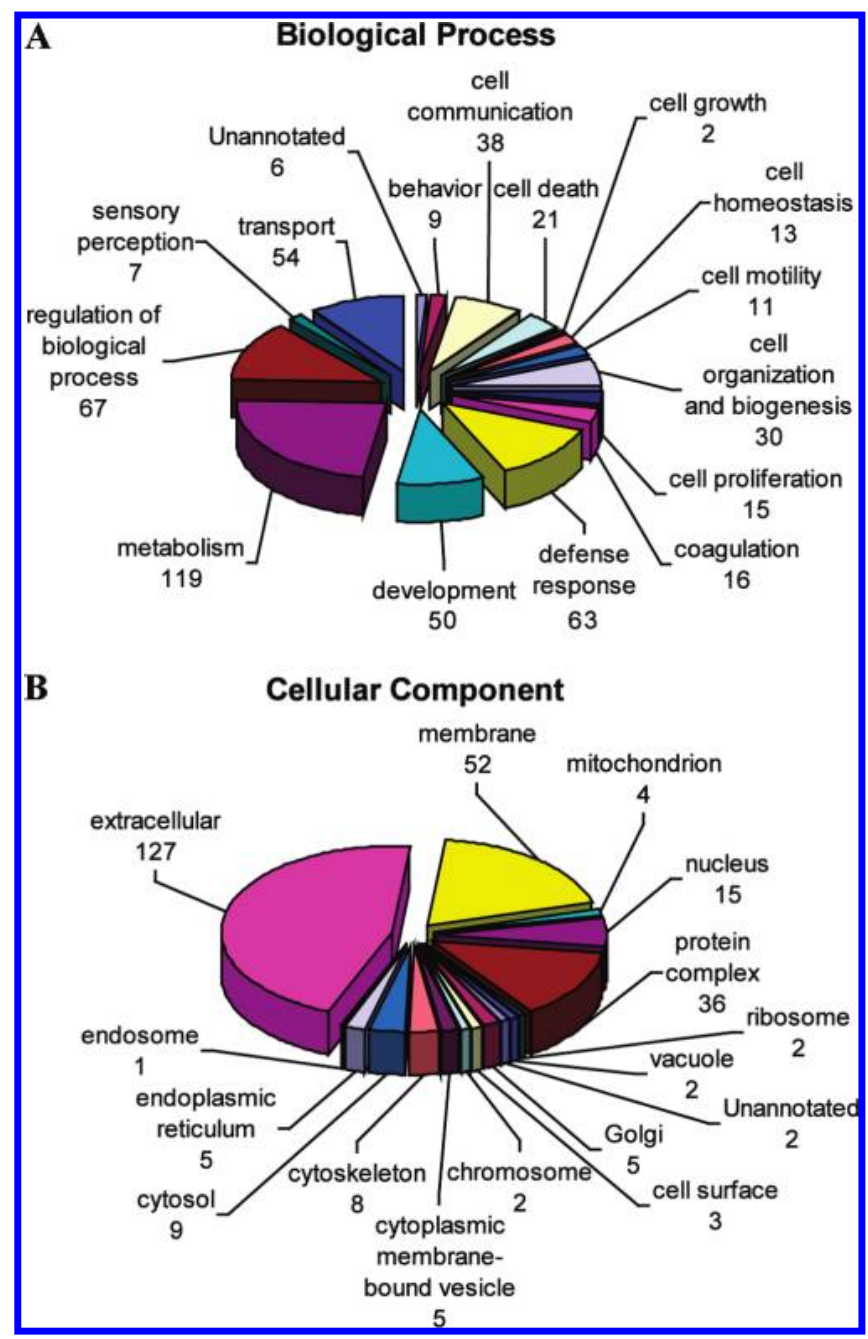

Figure 4. Classification of the proteins identified in PCLD cyst fluid. A total of 163 proteins were identified from a pool of 5 PCLD cyst fluids using one-dimensional SDS-PAGE gel electrophoresis followed by $\mathrm{nLC}-\mathrm{MS} / \mathrm{MS}$. Proteins were categorized according to their Gene Ontology terms for $(A)$ involvement in biological processes and (B) predicted cellular localization.

Western blot analysis with immunostaining for hepatocystin on non-albumin-depleted samples ( $n=6$, data not shown). Once again, hepatocystin was not detected. Altogether, these data suggest that hepatocystin is not secreted in liver cyst fluid. This indicates that hepatocystin is either contained in the cells lining the cysts or is not produced at all. Recently, we have shown that hepatocystin is absent in cyst epithelia of patients harboring a truncating mutation in hepatocystin. ${ }^{15}$ Thus, it seems that despite the dominant inheritance pattern of the disease, hepatocystin is not produced in cyst epithelia. Further research is needed to clarify the mechanisms involved. One of the possibilities is that mutated PRKCSH mRNA from cyst lining is subjected to nonsense mediated RNA decay (reviewed in ref 16).

In 1982, Patterson et al. described the biochemical characteristics of two liver cyst fluid samples from a single PCLD patient. ${ }^{17}$ In line with our results, they found a serum like protein pattern and identified $\alpha$-1-antitrypsin, $\alpha$-2-macroglobulins, hepatoglobin, albumin, transferrin and poly IgG and IgA in the fluids. Several studies have shown that ADPKD kidney cyst fluid contain cytokines and growth factors both of which contribute to cyst formation and cyst growth when imposed on kidney cell lines or cells from ADPKD cyst epithelium. ${ }^{18-22}$ We did not observe cytokines or growth factors in our samples, but we did not use specific enzyme-linked immunosorbent assays in order to detect these molecules. The Gene Ontology classification of the proteins identified by nLC-MS/MS showed that most proteins we identified are involved in metabolism $(73 \%)$, regulation of biological processes (41\%), and defense response (39\%). Only 9\% of proteins identified play a role in either stimulation or inhibition of cell proliferation. However, apart from proteins, other biological factors present in the cyst fluids, such as cAMP or lipids, may contribute to cystogenesis. ${ }^{23-25}$

\section{Conclusion}

The results presented here demonstrate that hepatocystin is not secreted in liver cyst fluid from PRKCSH mutated PCLD patients, despite the central role of hepatocystin in PCLD. This suggests mutant hepatocystin is either not produced or degraded intracellularly. We have identified a large number of ubiquitous serum proteins in liver cyst fluid, even though PCLD cysts develop from the intralobular bile ducts. Many of the identified proteins fulfill a role in metabolism and paracrine or autocrine proliferation factors were not recognized. Furthermore, PCLD cyst fluid resembles that of ADPKD and isolated liver cyst fluid indicating a common pathway for cystogenesis.

Acknowledgment. The authors thank all the patients for their participation in our study and Hans J. C. T. Wessels for his bioinformatics assistance with PROVALT. Joost P. H. 
Drenth is supported by a VIDI fellowship from The Netherlands Organization for Scientific Research (NWO).

Supporting Information Available: Supplementary Table 1 lists the proteins identified by nLC-MS/MS. The first hit in each group is highlighted in red, likely contaminants are listed in gray. This material is available free of charge via the Internet at http://pubs.acs.org.

\section{References}

(1) Qian, Q.; Li, A.; King, B. F.; Kamath, P. S.; Lager, D. J.; Huston, J.; Shub, C.; Davila, S.; Somlo, S.; Torres, V. E. Clinical profile of autosomal dominant polycystic liver disease. Hepatology 2003, 37 (1), 164-171.

(2) Gabow, P. A.; Johnson, A. M.; Kaehny, W. D.; Manco-Johnson, M. L.; Duley, I. T.; Everson, G. T. Risk factors for the development of hepatic cysts in autosomal dominant polycystic kidney disease. Hepatology 1990, 11 (6), 1033-1037.

(3) Drenth, J. P.; Te Morsche, R. H.; Smink, R.; Bonifacino, J. S.; Jansen, J. B. Germline mutations in PRKCSH are associated with autosomal dominant polycystic liver disease. Nat. Genet. 2003, 33 (3), 345347.

(4) Li, A.; Davila, S.; Furu, L.; Qian, Q.; Tian, X.; Kamath, P. S.; King B. F.; Torres, V. E.; Somlo, S. Mutations in PRKCSH cause isolated autosomal dominant polycystic liver disease. Am. J. Hum. Genet. 2003, 72 (3), 691-703.

(5) Trombetta, E. S.; Fleming, K. G.; Helenius, A. Quaternary and domain structure of glycoprotein processing glucosidase II. Biochemistry 2001, 40 (35), 10717-10722.

(6) Drenth, J. P.; Martina, J. A.; van de, Kerkhof, R.; Bonifacino, J. S.; Jansen, J. B. Polycystic liver disease is a disorder of cotranslational protein processing. Trends Mol. Med. 2005, 11 (1), 37-42.

(7) Drenth, J. P.; Martina, J. A.; Te Morsche, R. H.; Jansen, J. B.; Bonifacino, J. S. Molecular characterization of hepatocystin, the protein that is defective in autosomal dominant polycystic liver disease. Gastroenterology 2004, 126 (7), 1819-1827.

(8) Ravine, D.; Gibson, R. N.; Walker, R. G.; Sheffield, L. J.; KincaidSmith, P.; Danks, D. M. Evaluation of ultrasonographic diagnostic criteria for autosomal dominant polycystic kidney disease 1. $\underline{\text { Lancet }}$ 1994, 343 (8901), 824-827.

(9) Waanders, E.; Te Morsche, R. H.; de Man, R. A.; Jansen, J. B.; Drenth, J. P. Extensive mutational analysis of PRKCSH and SEC63 broadens the spectrum of polycystic liver disease. Hum. Mutat. 2006, 27 (8), 830.

(10) Pappin, D. J.; Hojrup, P.; Bleasby, A. J. Rapid identification of proteins by peptide-mass fingerprinting. Curr. Biol. 1993, 3 (6), 327-332.

(11) Ochs, D. Protein contaminants of sodium dodecyl sulfate-polyacrylamide gels. Anal. Biochem. 1983, 135 (2), 470-474.

(12) Rappsilber, J.; Friesen, W. J.; Paushkin, S.; Dreyfuss, G.; Mann, M. Detection of arginine dimethylated peptides by parallel precursor ion scanning mass spectrometry in positive ion mode. Anal. Chem. 2003, 75 (13), 3107-3114.
(13) Weatherly, D. B.; Astwood, J. A.; Minning, T. A.; Cavola, C.; Tarleton, R. L.; Orlando, R. A Heuristic method for assigning a false-discovery rate for protein identifications from Mascot database search results. Mol. Cell. Proteomics 2005, 4 (6), 762-772.

(14) Ashburner, M.; Ball, C. A.; Blake, J. A.; Botstein, D.; Butler, H.; Cherry, J. M.; Davis, A. P.; Dolinski, K.; Dwight, S. S.; Eppig, J. T.; Harris, M. A.; Hill, D. P.; Issel-Tarver, L.; Kasarskis, A.; Lewis, S.; Matese, J. C.; Richardson, J. E.; Ringwald, M.; Rubin, G. M.; Sherlock, G. Gene ontology: tool for the unification of biology. The Gene Ontology Consortium. Nat. Genet. 2000, 25 (1), 25-29.

(15) Waanders, E.; Croes, H. J.; Maass, C. N.; Te Morsche, R. H.; van Geffen, H. J.; van Krieken, J. H.; Fransen, J. A.; Drenth, J. P. Cysts of PRKCSH mutated polycystic liver disease patients lack hepatocystin but express Sec63p. Histochem. Cell Biol. 2008, 129 (3), 301-310.

(16) Weischenfeldt, J.; Lykke-Andersen, J.; Porse, B. Messenger RNA surveillance: neutralizing natural nonsense. Curr. Biol. 2005, 15 (14), R559-R562.

(17) Patterson, M.; Gonzalez-Vitale, J. C.; Fagan, C. J. Polycystic liver disease: a study of cyst fluid constituents. Hepatology 1982, 2 (4), $475-478$.

(18) Li, A.; Dubey, S.; Varney, M. L.; Dave, B. J.; Singh, R. K. IL-8 directly enhanced endothelial cell survival, proliferation, and matrix metalloproteinases production and regulated angiogenesis. J. Immunol. 2003, 170 (6), 3369-3376.

(19) Munemura, C.; Uemasu, J.; Kawasaki, H. Epidermal growth factor and endothelin in cyst fluid from autosomal dominant polycystic kidney disease cases: possible evidence of heterogeneity in cystogenesis. Am. J. Kidnev Dis. 1994, 24 (4), 561-568.

(20) Nichols, M. T.; Gidey, E.; Matzakos, T.; Dahl, R.; Stiegmann, G.; Shah, R. J.; Grantham, J. J.; Fitz, J. G.; Doctor, R. B. Secretion of cytokines and growth factors into autosomal dominant polycystic kidney disease liver cyst fluid. Hepatology 2004, 40 (4), 836-846.

(21) Slade, M. J.; Kirby, R. B.; Pocsi, I.; Jones, J. K.; Price, R. G. Presence of laminin fragments in cyst fluid from patients with autosomal dominant polycystic kidney disease (ADPKD): role in proliferation of tubular epithelial cells. Biochim. Biophys. Acta 1998, 1401 (2), 203-210.

(22) Ye, M.; Grant, M.; Sharma, M.; Elzinga, L.; Swan, S.; Torres, V. E.; Grantham, J. J. Cyst fluid from human autosomal dominant polycystic kidneys promotes cyst formation and expansion by renal epithelial cells in vitro. J. Am. Soc. Nephrol. 1992, 3 (4), 984-994.

(23) Grantham, J. J.; Ye, M.; Davidow, C.; Holub, B.; Sharma, M. Evidence for a potent lipid secretagogue in the cyst fluids of patients with autosomal dominant polycystic kidney disease. I. Am. Soc. Nephrol. 1995, 6 (4), 1242-1249.

(24) Mangoo-Karim, R.; Uchic, M. E.; Grant, M.; Shumate, W. A.; Calvet, J. P.; Park, C. H.; Grantham, J. J. Renal epithelial fluid secretion and cyst growth: the role of cyclic AMP. FASEB J. 1989, 3 (14), 2629-2632.

(25) Masyuk, T. V.; Masyuk, A. I.; Torres, V. E.; Harris, P. C.; Larusso, N. F. Octreotide inhibits hepatic cystogenesis in a rodent model of polycystic liver disease by reducing cholangiocyte adenosine 3',5'-cyclic monophosphate. Gastroenterology 2007, 132 (3), 1104-1116.

PR8000282 